\title{
Mass-to-Light Ratios of Early- and Late-Type Galaxies
}

\author{
Tereasa G. Brainerd \& Michael A. Specian \\ Boston University, Institute for Astrophysical Research, 725 \\ Commonwealth Ave., Boston, MA, USA, 02215
}

\begin{abstract}
Using the 100k data release of the 2dF Galaxy Redshift Survey (2dFGRS), we obtain mass-to-light ratios for isolated "host" galaxies via the dynamics of satellite galaxies which orbit about them. Distinct differences are seen in the dependence of $M / L$ on host luminosity for early-type vs. late-type hosts.
\end{abstract}

\section{Summary}

We use the criteria set out by McKay et al. (2002) to select isolated host galaxies and their satellites from the $100 \mathrm{k}$ data release of the 2dFGRS. Host galaxies must be at least twice as luminous as any other galaxy which falls within a velocity difference of $|d v| \leq 1000 \mathrm{~km} \mathrm{~s}^{-1}$ and a projected radius of $2 h^{-1} \mathrm{Mpc}$. Satellites must be at least 4 times fainter than the host, must fall within a velocity difference of $|d v| \leq 1000 \mathrm{~km} \mathrm{~s}^{-1}$, and must lie within a projected radius of $500 h^{-1} \mathrm{kpc}$. In addition, a host galaxy's luminosity must exceed the sum total of the luminosities of its satellites. Further, we ignore all potential host galaxies which were visually classified by the 2dFGRS team to be galaxy-galaxy mergers, as these are unlikely to be dynamically relaxed systems. From this we obtain a sample of 75 elliptical hosts with 171 satellites, 84 S0 hosts with 303 satellites, and 243 spiral hosts with 478 satellites. The median redshift of the early-type (E/S0) hosts is 0.062 , and for the late-type hosts $z_{\text {med }}=0.055$.

We compute the masses of the hosts interior to $r=260 h^{-1} \mathrm{kpc}$ using

$$
M(r)=-\frac{r \sigma_{v}^{2}}{G} \frac{\partial \ln \rho}{\partial \ln r}
$$

where $\sigma_{v}^{2}$ is the line of sight velocity dispersion of the satellites and $\rho(r)$ is the number density of satellites (e.g., McKay et al. 2002). Values of $\sigma_{v}$ are computed by fitting a Gaussian profile plus a constant offset to the distribution of host-satellite velocity differences, $|d v|$. The offset accounts for interloper galaxies which introduce a random component to the distribution of $|d v|$. The inferred values of $\sigma_{v}$ are independent of projected radius; however, the fraction of interlopers increases from $\sim 10 \%$ for $r \lesssim 0.1 h^{-1} \mathrm{kpc}$ to $\sim 35 \%$ for $0.4 h^{-1} \mathrm{kpc} \lesssim r \lesssim 0.5 h^{-1} \mathrm{kpc}$. The number density of satellites is $\rho_{E}(r) \propto r^{-2.11 \pm 0.09}$ (early-type hosts) and $\rho_{L}(r) \propto r^{-2.2 \pm 0.1}$ (late-type hosts).

The luminosities of the host galaxies are computed assuming a flat, $\Lambda$ dominated cosmology $\left(\Omega_{0}=0.3, \Lambda_{0}=0.7\right)$, for which the absolute magnitude of an $L^{*}$ galaxy in the $b_{J}$ band is $M_{b_{J}}^{*}-5 \log _{10} h=-19.66 \pm 0.07$ (Norberg et 


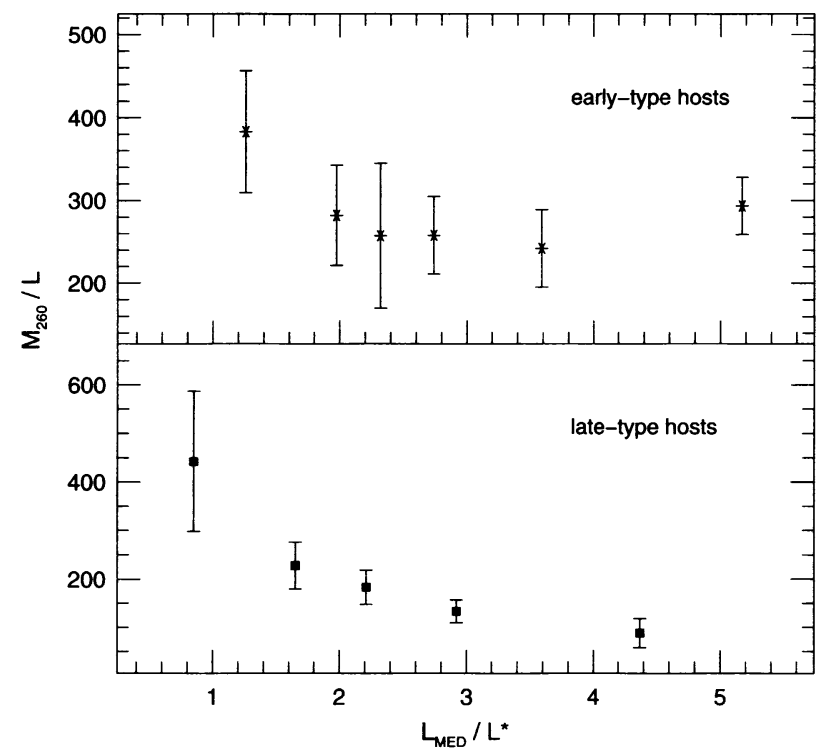

Figure 1. Mass-to-light ratios for the host galaxies interior to a radius of $260 h^{-1} \mathrm{kpc}$.

al. 2002). In the case of the early-type hosts, there is a clear increase of $\sigma_{v}$ with host luminosity: $\sigma_{v}=(43 \pm 7) L / L^{*}+(149 \pm 22) \mathrm{km} \mathrm{s}^{-1}$. In sharp contrast to this, the velocity dispersion of the satellites surrounding the late-type hosts is independent of the host luminosity: $\sigma_{v}=189 \pm 9 \mathrm{~km} \mathrm{~s}^{-1}$.

Shown in Fig. 1 is the dependence of $M_{260} / L$ on host luminosity for the early- and late-type hosts. For $L \gtrsim 2 L^{*}$, the mass-to-light ratios of the earlytype hosts is roughly constant with a value of $271 \pm 26 h M_{\odot} / L_{\odot}$. This is in good agreement with the mass-to-light ratios of early-type galaxies found in the weak lensing analysis of SDSS galaxies by McKay et al. (2001). The mass-to-light ratios of the late-type hosts in our sample, however, decrease monotonically with host luminosity: $M_{260} / L \propto L^{-1.0 \pm 0.2}$. The monotonic decrease is, of course, due to the fact that $\sigma_{v}$ appears to be independent of host luminosity in our sample of late type hosts and, while this conflicts with the lensing results of McKay et al. (2001), it is consistent with the dynamical results of Zaritsky et al. (1997), who found that the velocity difference between 69 isolated spirals and 115 satellites was independent of the host luminosity.

This work was supported by NSF contract AST 00-98572.

\section{References}

McKay, T. A., et al. 2001 (astro-ph/0108013)

McKay, T. A., et al. 2002, ApJ, 571, L85

Norberg, P., et al. 2002, MNRAS, 336, 907

Zaritsky, D., Smith, R., Frenk, C. \& White, S. D. M. 1997, ApJ, 478, 39 